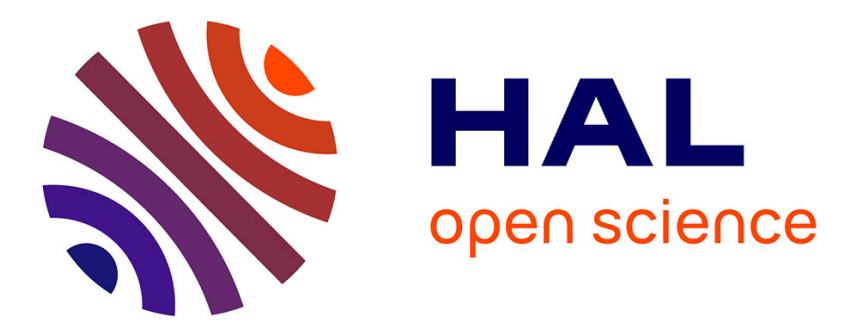

\title{
Influence of the inclination of a plate on forces generated in flows of Newtonian and yield stress fluids
}

\author{
Ziemihori Ouattara, Albert Magnin, Didier Blésès, Pascal Jay
}

\section{To cite this version:}

Ziemihori Ouattara, Albert Magnin, Didier Blésès, Pascal Jay. Influence of the inclination of a plate on forces generated in flows of Newtonian and yield stress fluids. Chemical Engineering Science, 2019, 197, pp.246-257. 10.1016/j.ces.2018.12.026 . hal-02412963

\section{HAL Id: hal-02412963 \\ https://hal.science/hal-02412963}

Submitted on 21 Oct 2021

HAL is a multi-disciplinary open access archive for the deposit and dissemination of scientific research documents, whether they are published or not. The documents may come from teaching and research institutions in France or abroad, or from public or private research centers.
L'archive ouverte pluridisciplinaire HAL, est destinée au dépôt et à la diffusion de documents scientifiques de niveau recherche, publiés ou non, émanant des établissements d'enseignement et de recherche français ou étrangers, des laboratoires publics ou privés.

\section{(c) (1) $\$$}

Distributed under a Creative Commons Attribution - NonCommercial| 4.0 International 


\title{
Influence of the inclination of a plate on forces generated in flows of Newtonian and yield stress fluids
}

\author{
Ziemihori Ouattara, Albert Magnin, Didier Blésès, Pascal Jay* \\ Université Grenoble Alpes, LRP, F-38000 Grenoble, France \\ CNRS, LRP, F-38000 Grenoble, France
}

\section{Abstract}

The aim of this article is to study experimentally and numerically the influence of the inclination of a plate placed near a wall on flows of Newtonian and yield stress fluids. The drag and lift forces were particularly studied. The inclination of the plate varies between $0^{\circ}$ and $90^{\circ}$. The inertia of the fluid is considered negligible. The effects of the Oldroyd number, the shear-thinning index and the inclination of the plate on drag and lift are shown. Experimentally, an instrumented flume was used with a Carbopol gel as a yield stress fluid model. The measured lift and drag forces are compared to the numerical results and the literature data. The elasticity and plasticity effects are discussed.

Key words: Yield stress fluid, Newtonian fluid, Inclined plate, Drag, Lift

*Corresponding author: pascal.jay@univ-grenoble-alpes.fr 


\section{Introduction}

Many industrial systems involve flows around fixed or mobile obstacles of various geometries and in particular plates. For example, flows in mixers or exchangers which are widely used in chemical engineering. These must be properly designed, and this cannot be achieved without a good understanding of the interactions. The creeping flow around a plate has been widely studied for Newtonian fluids. However, in the domain of non-Newtonian fluids and, in particular, in the case of yield stress fluids, very few studies seem to exist. To our knowledge, the determination of the forces generated on a plate in a yield stress fluid as a function of its inclination angle has never been studied both experimentally and numerically. For this flow configuration and for a Newtonian fluid, In et al. ${ }^{1}$ carried out a $2 \mathrm{D}$ numerical study of flow around a flat plate with a variable incidence angle and Reynolds numbers varying between 1 and 30. They calculated drag and lift coefficients as a function of plate angle. In addition, several studies were carried out for the flow of a Newtonian fluid parallel ${ }^{2,3}$ and perpendicular ${ }^{4,5}$ to the plate in the infinite domain.

On the other hand, for a power-law fluid or shear-thinning fluid, $\mathrm{Wu}$ and Thompson ${ }^{6}$ performed numerical and experimental studies of flow around an inclined plate. The effects of Reynolds number, shear-thinning index and angles of attack on drag and lift coefficients of the flat plate were presented.

For yield stress fluids, the only studies carried out to our knowledge concern the configurations for which the plate is perpendicular or parallel to the flow. For the flow of viscoplastic fluids parallel to the plate, an analytical study was performed by Piau ${ }^{7}$. He proposed new approaches for determining the kinematic field and stresses for the boundary layer along the plate for creeping flows. Piau et Debiane ${ }^{8}$ analyzed the variation in the drag coefficient and thickness of the boundary layer around a flat plate by describing the liquid region with a Herschel-Bulkley viscoplastic model and the solid region with Hooke's 
constitutive equation. Boujlel et al. ${ }^{9}$ studied experimentally the drag force and velocity fields around a plate parallel to the flow for a wide range of velocities. Balmforth et al. ${ }^{10}$ reexamined Oldroyd's viscoplastic boundary layer analysis as previously performed by Piau ${ }^{7}$. They proposed a general solution with an alternative theory that allows the boundary layer to be curved and of finite length. Contrary to Piau ${ }^{7}$, they found static and mobile rigid zones outside the boundary layer. In addition, Balmforth et al. ${ }^{10}$ indicated that it was not necessary to introduce elastic deformation below the yield stress to describe the displacement far from the plate, thus contradicting the studies of Boujlel et al. ${ }^{9}$. For the same configuration, Ahonguio et al. ${ }^{11}$ recently carried out a numerical and experimental study with an elastoviscoplastic model in which the variation in the plastic drag coefficient according to the Oldroyd numbers and the shear stress on the wall for various elastic numbers is determined. It should also be noted that the study of the effect of elasticity on the velocity profiles and the rigid zones around the plate in the flow has recently been studied numerically by Ferreira et al. ${ }^{12}$. For flows perpendicular to plates and knife-edges, Brookes and Whitmore ${ }^{13,14}$ studied experimentally the static drag force of these submerged bodies in China clay suspensions. However, the results are questionable because the rheometrical characterization of the clay suspensions is fragmentary and the slip on the walls of the obstacles is not controlled. Savreux et al. ${ }^{15}$ used a Bingham model for the numerical study of the flow structure with a negligible inertia as a function of the Oldroyd number. They proposed empirical solutions for the drag coefficient for Reynolds numbers ranging from 0.01 to 30 . In two recent articles, Patel and Chhabra ${ }^{16,17}$ carried out a numerical study of the flow of a Bingham fluid around an elliptical cylinder. In a first study ${ }^{16}$, they examined the influence of the aspect ratio. When the aspect ratio of the elliptical cylinder tends to low values, the case becomes similar to that of flow perpendicular to a plate. When the aspect ratio is high, the case becomes similar to that of a plate placed parallel to the flow. In a second work ${ }^{17}$, they studied the flow around a cylinder 
inclined with respect to the flow for an aspect ratio equal to 0.5 . These studies will be included in the comparisons analyzed below.

Studies related to the plasticity theory for applications in soil mechanics can also be found for plates perpendicular or parallel to the ultimate pull-out force. They are relatively numerous given the importance of applications. For example, Merifield et al. ${ }^{18}$ carried out a numerical study of the influence of the shape of the anchors on the holding capacity in clay over a range of depths and for varying widths and lengths. They calculated the values of the ultimate pullout forces at soil failure limits on the plate-shaped anchors. Bemben et al. ${ }^{19}$ carried out an experimental study of the effect of various loading conditions on the vertical holding capacity of marine anchors in sand and clay subjected to static and cyclic loading. They determined the values of the load holding capacity and the static breakout factor as a function of anchor embedment depth.

Unlike the previous works cited, this study will provide new knowledge of the inclination effects of the plate on drag and lift forces generated in the flow of a yield stress fluid and a Newtonian fluid. The plate will be completely immersed in the fluid. First, the problem and governing equations are described. The experimental approach will then be detailed with the rheological characterization of the fluids and experimental set-up. Forces acting on the plate will be measured in the Carbopol gel, which is considered to be a yield stress fluid model, and in a Newtonian glucose syrup. The forces generated on the plate will be determined as a function of the inclination angle for high Oldroyd numbers. The numerical study will then be presented. The Herschel-Bulkley model, regularized with the Papanastasiou approach, will be used. The effects of the shear-thinning index n, and the yield stress Od on the drag and lift coefficients, will be then determined. In the final part of this article, experimental and numerical results obtained will be compared with those of the literature. The effects of elasticity and plasticity will be discussed. 


\section{Theory}

The two-dimensional steady-state flow of a Newtonian fluid and a viscoplastic fluid around a plate inclined at various angles is considered. Figure 1 shows the geometry of the problem. The plate of width $\mathrm{L}$ and thickness e is immersed in the fluid. The ratio e/L of the plate is equal to 0.1 . The fluid velocity $\mathrm{U}$ is uniform at the inlet. Dimensions of the domain are provided in Figure 1. The flat plate is at a symmetrical distance $\mathrm{H}$ between the upper wall (Wall 1) and the lower wall (Wall 2). The Wall 1 corresponds to the free surface and the wall 2 to the bottom of the flume. The minimum gap equal to $\mathrm{H}=3 \mathrm{~L}$ was chosen between the bottom of the flume (Wall 2) and the plate as in the experimental condition. A distance of $\mathrm{H}=3 \mathrm{~L}$ was taken between the plate and the upper wall 1 in the numerical simulation. Experimentally, this distance varies between $2 \mathrm{~L}$ and $3 \mathrm{~L}$ with the angle of inclination. The overall distance of the domain between the upper wall 1 and the lower wall 2 is $6 \mathrm{~L}$.

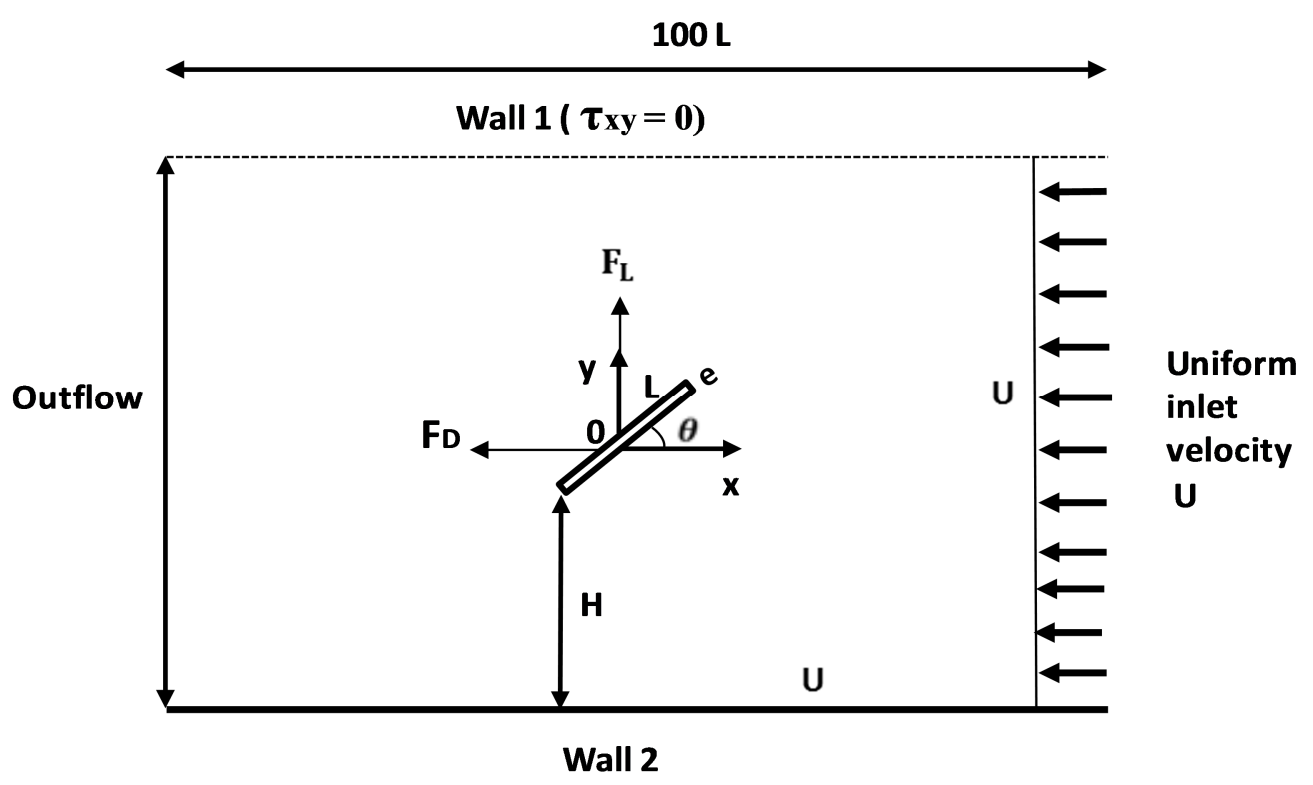

Figure 1: Schematic representation of the problem

The Herschel-Bulkley model used for the viscoplastic fluid behavior is expressed by: 


$$
\begin{cases}\underline{\underline{\tau}}=\left(\mathrm{K} \dot{\gamma}^{(\mathrm{n}-1)}+\frac{\tau_{0}}{\dot{\gamma}}\right) \underline{\dot{\gamma}} & \text { if } \tau>\tau_{0} \\ \underline{\underline{\underline{\gamma}}}=0 & \text { if } \tau \leq \tau_{0}\end{cases}
$$

where $K$ represents the consistency coefficient, $\tau_{0}$ the yield stress, $n$ the shear-thinning index, $\underline{\underline{\underline{\gamma}}}$ the tensor of the strain rates, $\dot{\gamma}$ the magnitude of the shear rate, $\underline{\underline{\tau}}$ the stress tensor, and $\tau$ the second invariant of the stress tensor. The yield surface is defined by the von Mises criterion. Ovarlez et al. ${ }^{20}$ and Martinie et al. ${ }^{21}$ have demonstrated that the yield stress that will be used in the experiments complies with the von Mises criterion.

The boundary conditions may be written as follows:

- At the inlet and on the wall 2: $\mathrm{U}_{\mathrm{x}}=\mathrm{U}$ and $\mathrm{U}_{\mathrm{y}}=0$

- On the wall 1: $\tau_{\mathrm{xy}}=0$

- On the plate: condition of no-slip: $\mathrm{U}_{\mathrm{x}}=0$ and $\mathrm{U}_{\mathrm{y}}=0$

- At the outlet, an outflow condition is defined as $\forall(\mathrm{i}, \mathrm{j}) \frac{\partial \mathrm{U}_{\mathrm{i}}}{\partial \mathrm{x}_{\mathrm{j}}}=0$

The following dimensionless parameters can be defined:

The shear-thinning index:

$\mathrm{n}$

The Oldroyd number (yield stress effect/viscous effect): $\quad \mathbf{O d}=\frac{\boldsymbol{\tau}_{\mathbf{0}}}{\mathbf{K}(\mathbf{U} / \mathbf{L})^{\mathbf{n}}}$

The Reynolds number (inertia effect/viscous effect):

$\operatorname{Re}=\frac{\rho L^{\mathrm{n}} \mathbf{U}^{2-n}}{\mathrm{~K}}$

The dimensionless minimum gap:

$$
\mathbf{G}=\frac{\mathbf{H}}{\mathbf{L}}
$$

The viscous and plastic drag and lift coefficients are defined by the following equations

$$
C d_{\text {vis }}=\frac{2 F_{\mathrm{D}} \cdot \mathrm{L}^{\mathrm{n}-1} \cdot \mathrm{U}^{-\mathrm{n}}}{\mathrm{K}}, \mathrm{Cd}^{*}=\frac{\mathrm{Cd}_{\mathrm{vis}}}{\mathrm{Od}}
$$




$$
\mathrm{Cl}_{\mathrm{vis}}=\frac{2 \mathrm{~F}_{\mathrm{p}} \cdot \mathrm{L}^{\mathrm{n}-1} \cdot \mathrm{U}^{-\mathrm{n}}}{\mathrm{K}}, \quad \mathrm{Cl}^{*}=\frac{\mathrm{Cl}_{\mathrm{vis}}}{\mathrm{Od}}
$$

$F_{D}$ and $F_{P}$ represent respectively the drag and lift forces per unit length. These are the forces exerted by the fluid on the obstacle. In the Cartesian coordinate system used in Figure1, the drag force is negative, and its value will be given as an absolute value. The lift force is positive.

\section{Experimental study}

\subsection{Rheological characterization}

A glucose syrup produced by Nigay S.A. with a density of $1500 \mathrm{~kg} / \mathrm{m}^{3}$ was used as a Newtonian fluid. The glucose viscosity was measured with shear rates ranging from 0.03 to $0.1 \mathrm{~s}^{-1}$ in a cone-plane geometry using a DHR3 rheometer (TA Instruments). An antievaporation cell around the sample was used. The viscosity was measured at a temperature ranging from 18 to $25^{\circ} \mathrm{C}+/-0.5^{\circ} \mathrm{C}$ to evaluate accurately the value of the viscosity in the flume experiment. For example, the viscosity of glucose at $20.7^{\circ} \mathrm{C}$ is about 2186 Pa.s. The Carbopol gel used in this study is often considered as a yield stress fluid model in fluid mechanics experiments in particular thanks to its absence of significant thixotropy (Piau ${ }^{22}$, Dimitriou et al. $^{23}$ ) and its transparency. Here, it was obtained by diluting a mass quantity of 0.85 wt $\%$ of Carbopol 940 manufactured by Goodrich $^{24}$ in distilled water. The translucent acidic solution obtained was then neutralized by the addition of sodium hydroxide solution to obtain a transparent gel. Rheometrical characterization was analyzed under the steady state and oscillatory conditions. In steady state, the rheological properties of the gel were determined at rotational speed, temperature and evaporation controlled by simple shear measurements using the DHR3 rheometer. Samples were taken directly from the flume at the free surface and at the bottom of the flume. The measurements were taken with a cone and plane cell for shear rates ranging from $10^{-3}$ to $100 \mathrm{~s}^{-1}$. The surfaces of the cell were covered 
with rough paper to prevent slippage, as shown in the study by Magnin and Piau ${ }^{25,26}$ and Meeker et al. ${ }^{27}$. The cone used had a diameter of $50 \mathrm{~mm}$ with an angle of 0.035 radians. Parameter measurement uncertainties were estimated to be $7 \%$ for the yield stress fluid and $15 \%$ for glucose syrup.

Table 1 presents the rheological properties of the Carbopol gel. The parameter values of the Herschel-Bulkley model (eq.(1)) are obtained after fitting with rheometrical measurements. This table gives also the elastic modulus $G^{\prime}$ and the viscous modulus $G$ ' in the linear domain below the yield stress of the material. The strain $\gamma$ is the limit of the linear domain.

\begin{tabular}{|c|c|c|c|c|c|}
\hline$\tau_{0}(\mathrm{~Pa})$ & $\mathrm{K}\left({\mathrm{Pa} . \mathrm{s}^{\mathrm{n}}}\right)$ & $\mathrm{n}$ & $\gamma(\%)$ & $\mathrm{G}^{\prime}(\mathrm{Pa})$ & $\mathrm{G}{ }^{\prime}(\mathrm{Pa})$ \\
\hline 115 & 40.4 & 0.4 & 0.3 & 561 & 29 \\
\hline
\end{tabular}

Table 1: Rheological properties of Carbopol gel

The Carbopol gel behaves like an elasto-viscoplastic fluid as shown by $\mathrm{Piau}^{22}$, Dimitriou et al. $^{23}$ and Coussot et al. ${ }^{28}$. Ahonguio et al. ${ }^{29}$ showed that these gels may have a non-zero first difference of normal stresses. Yarin et al. ${ }^{30}$ and Balmforth et al. ${ }^{31,32}$ studied the elongation behavior of these gels. The fluidization or solid-liquid transition of Carbopol gels has been the subject of recent studies by Ovarlez et al..$^{20}$ and Martinie et al. ${ }^{21}$.

Several authors studied the creep behavior under the yield stress (Lidon et al. ${ }^{33}$, Caton and Baravian $^{34}$, Divoux et al. ${ }^{35}$, Divoux et al. ${ }^{36}$ ). From an experimental standpoint, Dimitriou et al. $^{23}$ introduced the isotropic kinematic hardening (IKH) model which describes the variation in yield stress during flow. The study of Fraggedakis et al. ${ }^{37}$ is interesting to read in terms of its analysis of the IKH concept and its application to numerical modeling.

\subsection{Experimental set up}


The experimental set-up is presented in Figure 2.

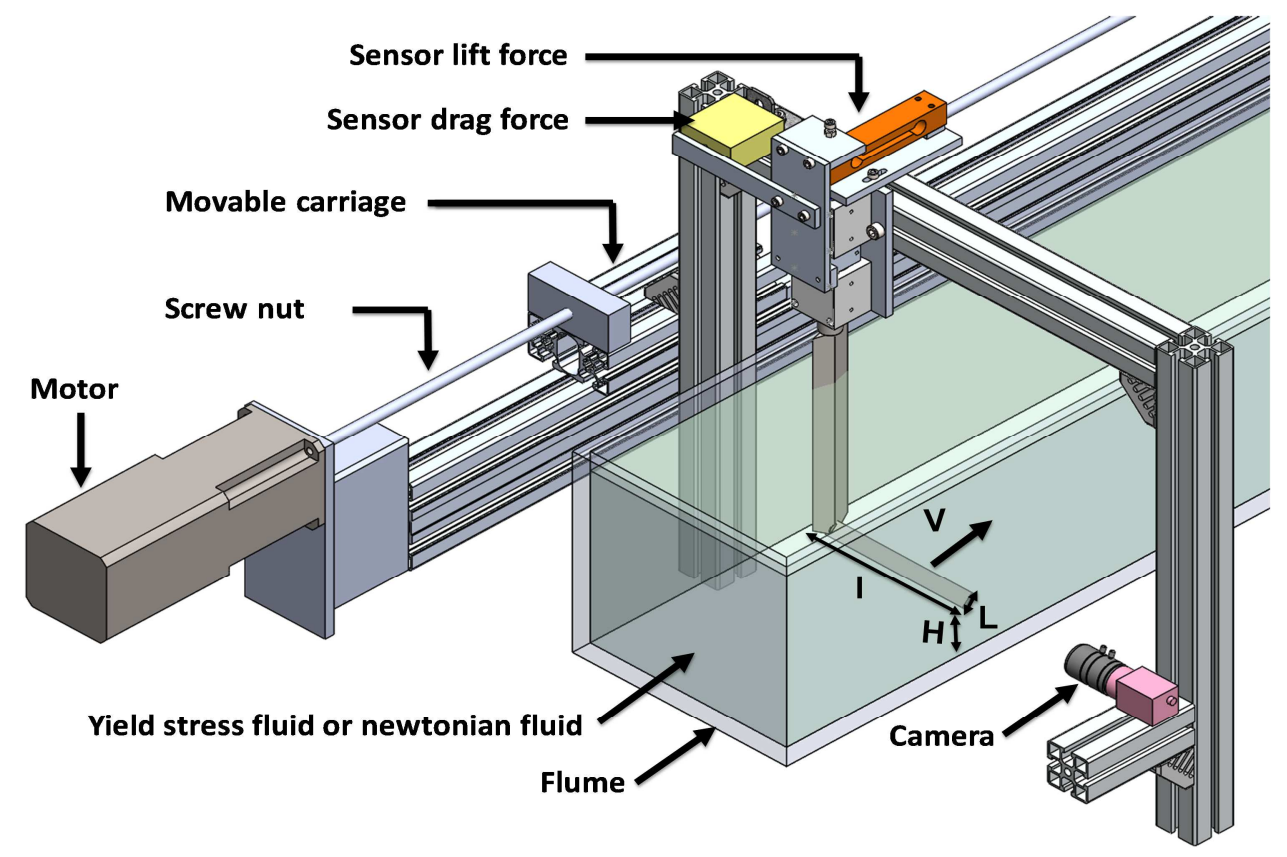

Figure 2: Experimental set-up

A movable carriage driven by a screw nut system rotated by a variable speed brushless motor moves the inclined plate and camera. The inclined plate is attached to drag and lift sensors. The applied velocity range is varied between 0.001 and $6 \mathrm{~mm} / \mathrm{s}$ with an accuracy of $4 \%$. The flume is made of transparent Plexiglas and is $3 \mathrm{~m}$ long, $22 \mathrm{~cm}$ wide and $21 \mathrm{~cm}$ high. The inclined plate is $14 \mathrm{~cm}+/-0.1 \mathrm{~mm}$ long, $3 \mathrm{~cm}+/-0.02 \mathrm{~mm}$ wide, and $0.3 \mathrm{~cm}+/-0.02 \mathrm{~mm}$ thick. To prevent slippage, the wall surface of the flume and the cylinder are coated with a sandpaper of average roughness $\mathrm{Ra}=120 \mu \mathrm{m}$ manufactured by Norton. Plate-to-wall distance is equal to $\mathrm{G}=3$. The minimum distance $\mathrm{H}$ between the plate and the bottom of the flume can be adjusted with an accuracy of $2 \%$. The sensor for measuring drag force was manufactured by TE Connectivity and the sensor for measuring the lift force by Scaime. These sensors can measure forces ranging from 0 to $50 \mathrm{~N}$ with an accuracy of about $0.05 \%$ of full scale for drag and $0.025 \%$ of full scale for lift. The parasitic drag force of the arm holding the plate was measured without the plate and for each velocity and gap value. The force due to the 
interaction between the plate tip and the vertical wall of the flume was also estimated for each velocity. These two parasitic forces were deducted from the measured value of the drag force with the plate in order to obtain only the drag force on the plate. It should be noted that the parasitic drag force of the plate holding arm without the plate is negligible and represents less than $2 \%$ of the overall value, and for lift, this value represents less than $3 \%$ of the overall value. The experimental uncertainties on the plastic drag and lift coefficients was $13 \%$ and $10 \%$ respectively. These uncertainties consist of $7 \%$ for yield stress, $6 \%$ for drag force and $3 \%$ for the lift force. Concerning the Oldroyd number, the uncertainty was of the order of $16 \%$.

During the measurements from rest, a transient state where the forces increase with time is observed, followed by a steady state independent of time. The duration of the transient state depends on the imposed velocity and nature of the fluid. At a fixed velocity, the transients in our experiments can be reproduced relatively. No evidence was found of phenomena such as those observed during sedimentation of spheres in Carbopol gels ${ }^{48,49}$. These authors ${ }^{48,49}$ pointed out that the reproducibility of the sphere terminal settling velocity is poor. Atapattu et al. $^{48}$ found that reproducible results could be obtained in Carbopol gels only after releasing 410 spheres. They found the settling velocity to be low for the first sphere. This velocity increases to an asymptotic value after a number of spheres were released. Hariharaputhiran et al. ${ }^{49}$ argue that these observations are consistent with the hypothesis of network damage caused by shear with subsequent healing. There are a number of differences between these flow conditions and ours. The present study is carried out with an imposed speed and not an imposed force as in settling. Our yield stress is higher by a factor of about 10 . Indeed, our suspension is more concentrated in Carbopol microgel and a more compact structure is thus generated $^{22}$. However, some tests have been done by changing the initial state of stress in the fluid. For example, a grid was passed through the fluid to homogenize the state of stress and 
the Carbopol gel structure. A notable difference in the transient of the first test was found compared to the transients obtained after the grid was passed. It is possible that this phenomenon is compatible with the observations made by the authors working on the settling of spheres in Carbopol but with less intensity. Similar phenomena of unusual transients and the effect of initial stress state were also observed on the very slow rise of bubbles in Carbopol gels ${ }^{47}$. Only steady state forces have been studied in depth in the present study.

\section{Experimental results}

\subsection{Newtonian fluid}

Figure 3 shows the variation in the drag and lift force as a function of velocity with various inclination angles for the glucose syrup. The Reynolds number varies between $2.68 \times$ $10^{-7}$ and $3.21 \times 10^{-5}$.
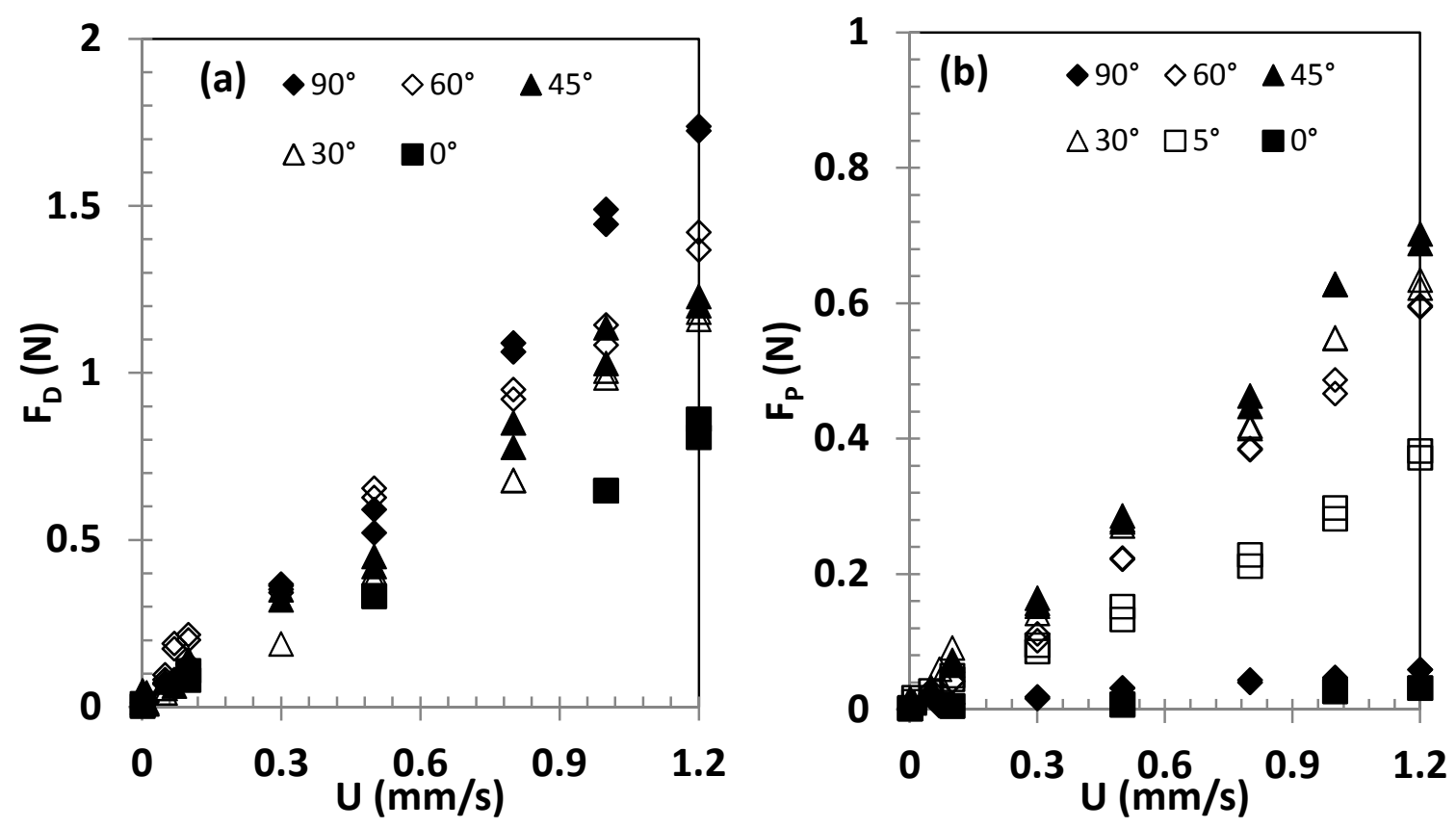

Figure 3: Variation in drag force (a) and lift (b) as a function of velocity for various inclination angles for a Newtonian fluid. 
It can be seen that lift and drag forces increase relatively linearly with the velocity for each angle. In Figure 3a, the drag force increases when the angle increases. In Figure $3 b$, the lift force is minimal at $0^{\circ}$ then increases with the angle up to approximately $45^{\circ}$, then decreases to zero at $90^{\circ}$. The lift values are non-zero at $0^{\circ}$ and $90^{\circ}$ angles for high velocities. This may be due to the non-negligible thickness of the plate and to the uncertainty surrounding the angle of inclination.

$\mathrm{Wu}$ and Thompson ${ }^{6}$ studied the flow of a non-Newtonian fluid around an inclined plane plate with a variable angle. They proposed analytical expressions for viscous drag $\mathrm{Cd}$ and lift $\mathrm{Cl}$ depending on the angle, defined by:

$$
\begin{aligned}
& \mathrm{Cd}=\mathrm{Cd}_{(\theta=90)} \sin ^{2} \theta+\mathrm{Cd}_{(\theta=0)} \cos ^{2} \theta \\
& \mathrm{Cl}=\frac{1}{2}\left(\mathrm{Cd}_{(\theta=90)}-\mathrm{Cd}_{(\theta=0)}\right) \sin 2 \theta
\end{aligned}
$$

They specified that these equations can also be applied to a shear-thinning fluid, but only in creeping flow. The drag and lift coefficients as a function of the angle can be calculated from the drag coefficient at $0^{\circ}\left(\mathrm{Cd}_{\mathrm{vis}(\theta=0)}\right)$ and $90^{\circ}\left(\mathrm{Cd}_{\mathrm{vis}(\theta=90)}\right)$ angles. These equations will be used in section 6 . 


\subsection{Yield stress fluid}

Figure 4 shows the variation in drag force (Fig.4a) and lift force (Fig. 4b) as a function of velocities for various inclination angles. The range of Reynolds number used is between $1.5 \times$ $10^{-9}$ and $1.7 \times 10^{-3}$ with Oldroyd numbers ranging from 5.5 to 179 . Figure 4 a shows that the variation in drag force as a function of velocity is similar, regardless of the inclination angle. Unlike Newtonian fluids, the drag force tends towards a plateau value at low velocities corresponding to high yield stress effects. The drag force increases with velocity because the viscous forces increase with respect to the yield stress forces. The level of force increases with the inclination angle. Figure $4 \mathrm{~b}$ shows that, just like the drag force, the lift force tends towards a plateau value when the velocity tends towards 0 and thus when the yield stress effects become high. The lift force increases with velocity when the velocity becomes higher.
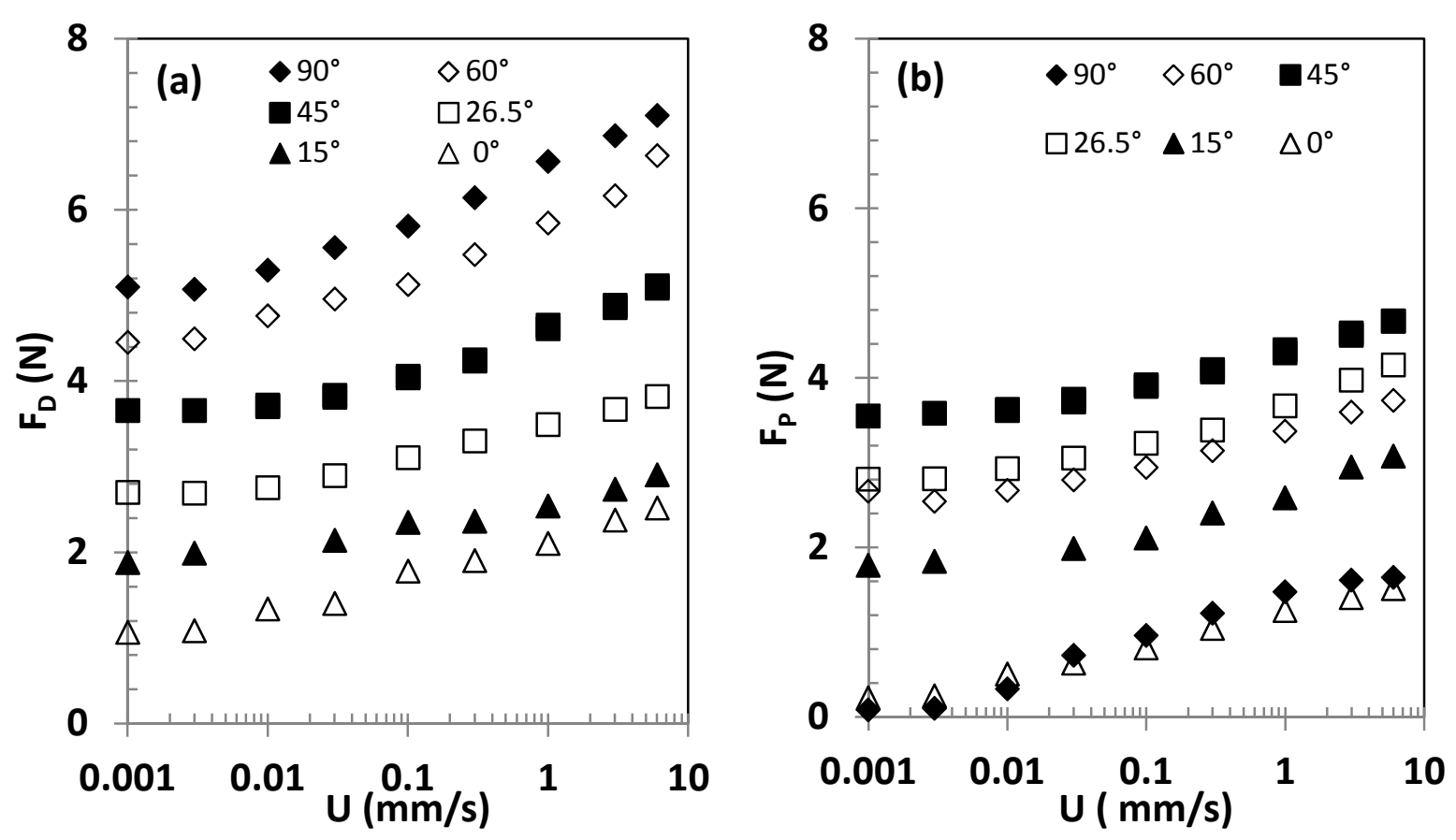

Figure 4: Variation in drag force (a) and lift (b) as a function of velocity for various plate inclination angles for the yield stress fluid. 
The lift force is minimum at $0^{\circ}$ and increases with the inclination angle. It is maximum at $45^{\circ}$ angle and then decreases as the angle increases. The lift values are non-zero at $0^{\circ}$ and $90^{\circ}$ angles for high velocities, like in the Newtonian fluid. The effect appears more pronounced than in the Newtonian fluid. The existence of these lift values at these angles may be due to the non-negligible thickness of the plate and to the rheological properties of the materials used.

Figure 5 presents the measurements results for the dimensionless representation. It shows the variation in plastic drag and lift coefficient as a function of the Oldroyd number for various inclination angles. Regardless of the inclination angle, these plastic coefficients decrease and tend towards a plateau value when the Oldroyd number becomes higher. Within this limit, the coefficients $\mathrm{Cd}^{*}$ and $\mathrm{Cl}^{*}$ depend only on the yield stress. On the other hand, as Oldroyd numbers decrease and viscous friction becomes higher, the coefficient values increase.

In the studies carried out by Merkak et al. ${ }^{38}$ and Tokpavi et al. ${ }^{39}$, the experimental data were interpolated by an empirical law defined by:

$\mathrm{Cd}^{*}=\mathrm{Cd}_{\infty}^{*}+\frac{\mathrm{B}_{1}}{\mathrm{Od}^{\mathrm{M}}}$ with $\mathrm{M}=1 /(1+\mathrm{n})$

$\mathrm{A}_{1}=\mathrm{Cd}_{\infty}^{*}$ represents the plastic contribution and $\mathrm{B}_{1}$ the viscous part. Similarly, the equation of the plastic lift coefficient can be defined as follows:

$\mathrm{Cl}^{*}=\mathrm{Cl}_{\infty}^{*}+\frac{\mathrm{B}_{2}}{\mathrm{Od}^{\mathrm{M}}}$

$\mathrm{A}_{2}=\mathrm{Cl}^{*}{ }_{\infty}$ is the plastic contribution of the lift and $\mathrm{B}_{2}$ represents the viscous contribution.

The values $\mathrm{Cd}^{*}{ }_{\infty}$ and $\mathrm{Cl}_{\infty}^{*}$ represent the plastic behavior of the material when Od tends towards infinity.

Figure 5 shows the variation in experimental values of the drag and lift coefficients according to the Oldroyd number and for various angles. These analytical expressions (10) and (11) are also shown in Figure 5. 

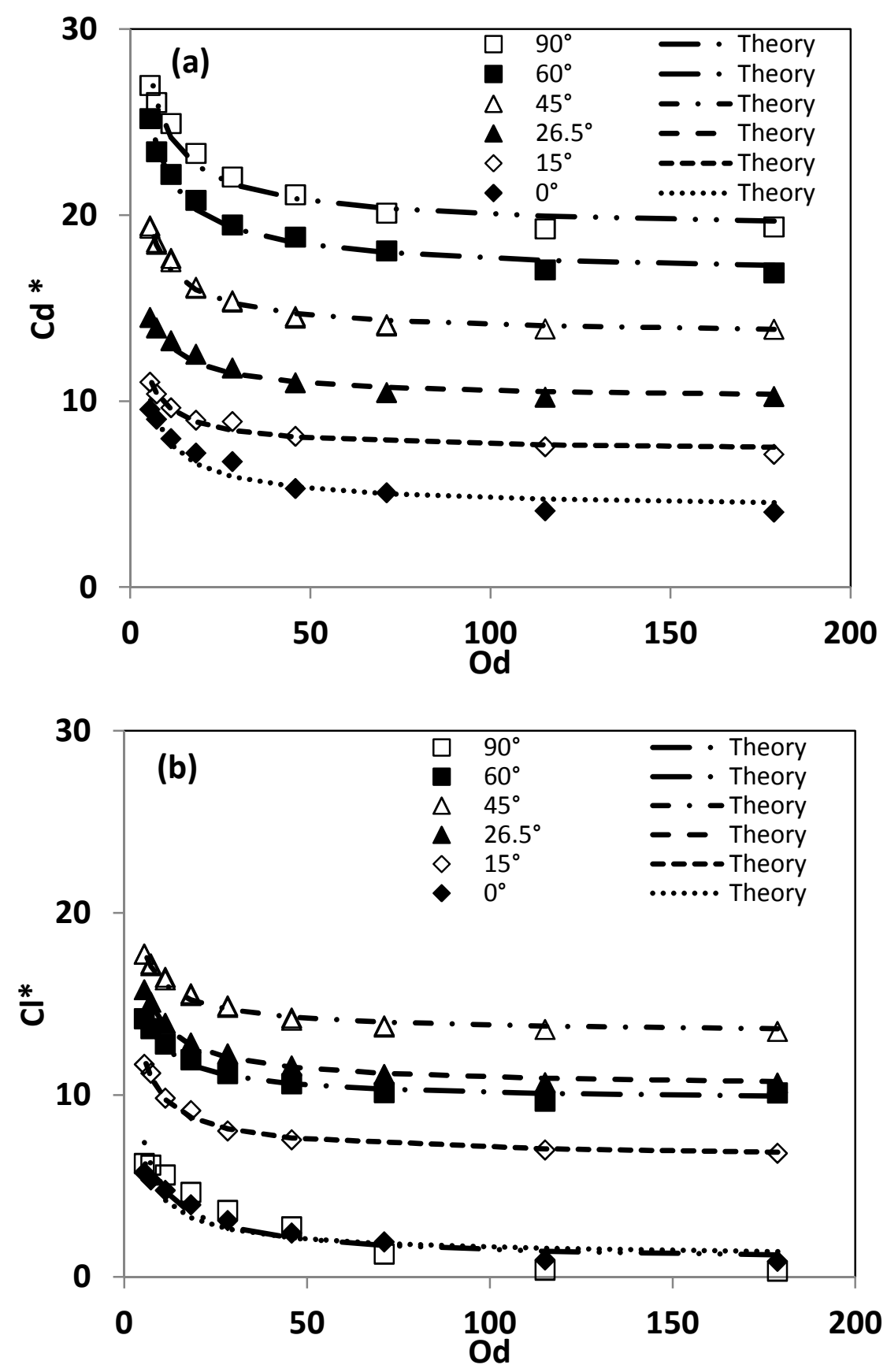

Figure 5: Variation in experimental plastic drag (a) and lift coefficient (b) as a function of the Oldroyd number for various inclination angles. The analytical solutions (eqs. (10) and (11)) are also plotted in the figure.

The values of the coefficients $A_{1}, A_{2}, B_{1}$ and $B_{2}$ are summarized in Table 2 as a function of angle. The value of $\mathrm{M}$ is equal to 0.714 in this study. 


\begin{tabular}{|c|c|c|c|c|}
\hline Parameters & \multicolumn{2}{|c|}{$\mathrm{Cd}^{*}$} & \multicolumn{2}{c|}{$\mathrm{Cl}^{*}$} \\
\hline$\Theta\left(^{\circ}\right)$ & $\mathrm{A}_{1}$ & $\mathrm{~B}_{1}$ & $\mathrm{~A}_{2}$ & $\mathrm{~B}_{2}$ \\
\hline 0 & 4.042 & 20.57 & 0.95 & 18.34 \\
\hline 15 & 7.197 & 13.45 & 6.4 & 18.89 \\
\hline 26.5 & 9.95 & 16.66 & 10.26 & 19.57 \\
\hline 45 & 13.41 & 20.11 & 13.3 & 15.12 \\
\hline 60 & 16.57 & 29.77 & 9.52 & 16.83 \\
\hline 90 & 18.96 & 29.54 & 0.64 & 22.84 \\
\hline
\end{tabular}

Table 2: Values of plastic drag $\mathrm{Cd}^{*}$ and lift $\mathrm{Cl}^{*}$ coefficient from eqs. (10) and (11)

The values of the analytical expressions found in Table 2 are valid for an Oldroyd number between 5.5 and 179 and a negligible inertia. It was found that the $\mathrm{A}_{1}$ values of the drag increase with the angle. The coefficient $\mathrm{A}_{2}$ increases with the angle up to a maximum of $45^{\circ}$ and then decreases.

On the basis of soil mechanics theory and, in particular, the calculation of anchors in a perfectly plastic soil (Raghavendra ${ }^{40}$, Aubeny and $\mathrm{Chi}^{41}$ ), the following expressions can also be written:

$$
\begin{aligned}
& \mathrm{Cd}^{*}=\mathrm{Cd}_{(\theta=90)}^{*} \sin ^{2} \theta+\mathrm{Cd}_{(\theta=0)}^{*} \cos ^{2} \theta \\
& \mathrm{Cl}^{*}=\frac{1}{2}\left(\mathrm{Cd}_{(\theta=90)}^{*}-\mathrm{Cd}_{(\theta=0)}^{*}\right) \sin 2 \theta
\end{aligned}
$$

Note that the maximum lift given by eq. (13) occurs at an angle of $\theta=45^{\circ}$. In addition, in their studies on plate anchoring, Raghavendra ${ }^{40}$ and Anderson et al. $^{42}$ proposed solutions for the drag $\mathrm{Cd}_{(\theta=0)}^{*}$ and $\mathrm{Cd}_{(\theta=90)}$ which are applicable to a perfectly plastic material.

These expressions are defined by the following eq. (14) and (15): 
$\mathrm{Cd}_{(\theta=0)}^{*}=4+30 \frac{\mathrm{e}}{\mathrm{L}}$

$\mathrm{Cd}_{(\theta=90)}^{*}=6 \pi+4+2 \frac{\mathrm{e}}{\mathrm{L}}\left(2+\frac{4}{\sqrt{2}}\right)$

Figure 6 compares the experimental results with those of this theory for high yield stress effects. Equations (14) and (15) give in our case: $\operatorname{Cd}_{(\theta=0)}^{*}=7$ and $\operatorname{Cd}_{(\theta=90)}^{*}=23.81$. Drag and lift were found to vary similarly with the theoretical results. In Figure 6a, the experimental values of the plastic drag and the theoretical prediction are generally close except at high angles. In Figure 6b, non-negligible differences were observed between the experimental values of lift and those of the plasticity theory of soil mechanics.
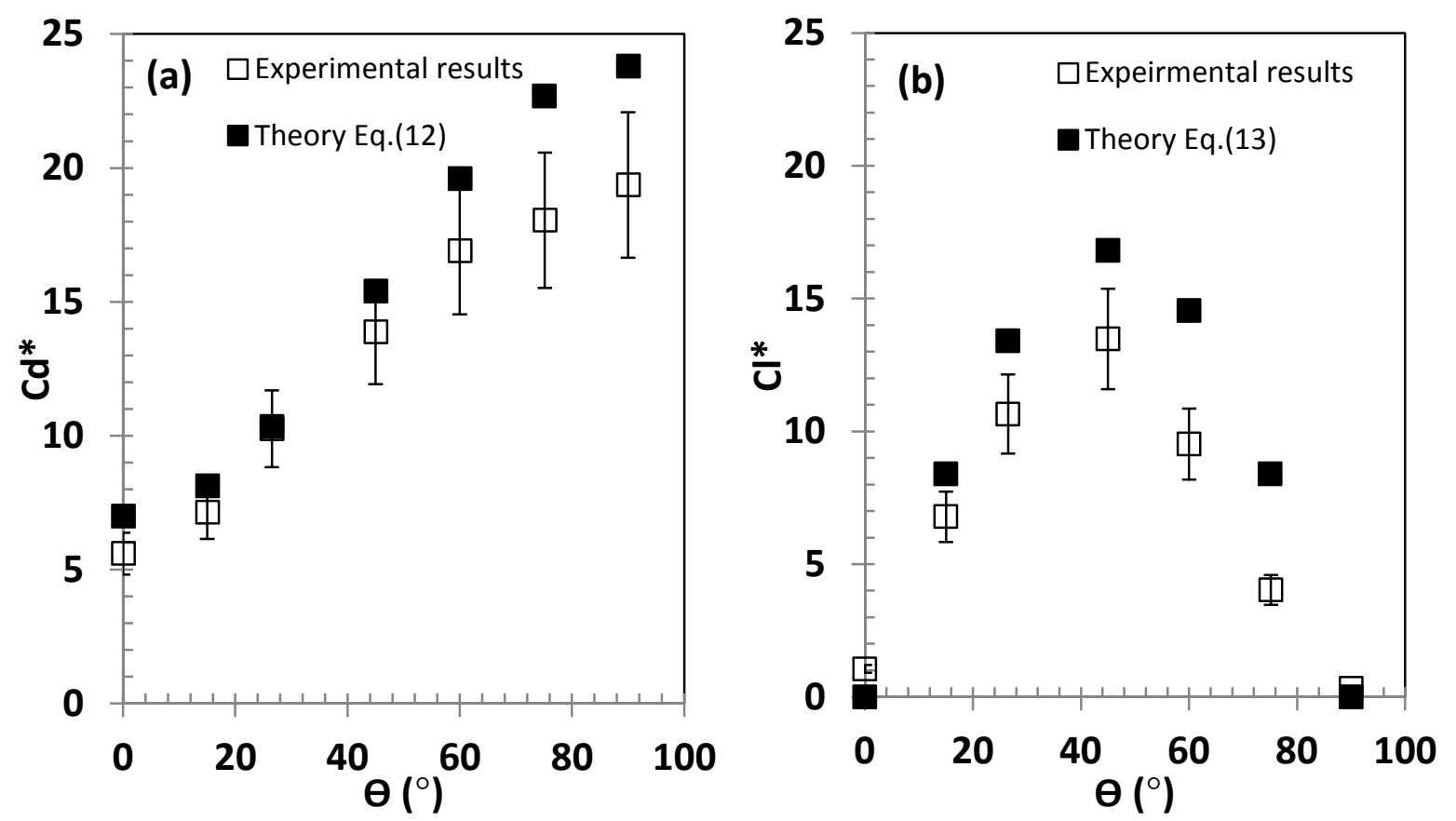

Figure 6: Comparative study between the values of the plastic drag and lift coefficient at Od= 179 with $n=0.4$ and the plasticity theory as a function of the inclination angle. 


\section{Numerical modeling}

A numerical study was performed to compare these predictions with the experimental results. For this modeling, the Herschel-Bulkley law (eq. (1)) was regularized by the wellknown Papanastasiou model ${ }^{43}$. This regularized model has already been used in many studies, particularly for flows around obstacles ${ }^{15,44,45}$. It is defined by the following eq. (16) where $\mathrm{m}$ represents the regularization parameter.

$$
\underline{\underline{\tau}}=\left[\mathbf{K} \dot{\boldsymbol{\gamma}}^{(\mathbf{n}-1)}+\frac{\tau_{0}\left(1-\exp ^{-\mathbf{m}|\dot{\gamma}|}\right)}{|\dot{\boldsymbol{\gamma}}|}\right] \underline{\underline{\boldsymbol{\gamma}}}
$$

\subsection{Numerical method}

Version 17.1 of the Ansys-Fluent software was used based on a finite volume approach with a double-precision solver and a second-order spatial discretization. The SIMPLEC (Semi-Implicit Method for Pressure Linked Equations-Consistent) method was adopted. An absolute convergence criterion of $10^{-8}$ was chosen for the convergence of velocity and continuity equations. For all the numerical simulations, the Reynolds number is kept very low at 0.001 .

\subsection{Influence of the mesh and the shape of the plate}

In order to optimize all the numerical parameters and to make the results independent of the numerical approach, a systematic study of the effect of the mesh (number of nodes), the shape of the plate (rectangular, oblong) and the type of mesh (quadrilaterals, triangles) on the plastic drag and lift coefficients was performed. For the mesh it was found that a minimal refinement of the order of 116000 nodes is required to make the mesh independent of the drag and lift coefficient. This mesh is highly refined towards the four corners of the plate 
The shape of the plate was also modified to verify whether the presence of corners, which may be singular points in the numerical modeling, had an effect on the value of the drag and lift coefficients. A plate with rounded edges was then created. The change in the geometric shape of the plate from a rectangle to that of a rounded shape does not modify the drag and the lift values to any great extent, with the maximum difference obtained being $5 \%$ for the $26.5^{\circ}$ angle and $5.5 \%$ for the $45^{\circ}$ angle. Singularities are present but they do not necessarily influence the values of force coefficients.

A triangular mesh was also made to verify that the type of mesh did not change the final result. Between the quadrilateral mesh and the triangular mesh a maximum difference of $3 \%$ was obtained.

The effect of varying the Reynolds number between 0.01 and 0.0001 on the drag and lift coefficients for the $45^{\circ}$ and $26.5^{\circ}$ angles was also studied. The values of these coefficients obtained in this range of Reynolds number are similar. Following this study, the refined quadrilateral mesh of 116000 nodes with a plate of rectangular shape will be used to carry out the numerical computations. For the Papanastasiou ${ }^{43}$ regularization (eq.16), the effect of the regularization parameter $\mathrm{m}$ on the force coefficients was also systematically studied and a value of $\mathrm{m}=10^{4}$ was chosen for all calculations. The boundary between yielded and unyielded zones is obtained by the condition $\tau=\tau_{0}(1 \pm \varepsilon)$ proposed by Burgos et al. ${ }^{50}$. The value of $\varepsilon=0$ was determined based on the study of Burgos et $\mathrm{al}^{50}$.

\subsection{Drag and lift coefficients}

In this part, shear-thinning fluids and then Herschel-Bulkley yield stress fluids will be examined.

\subsubsection{Shear-thinning fluid}

Figure 7 shows the variation in viscous drag and lift coefficients as a function of the angle at negligible inertia and for various values of the shear-thinning index $\mathrm{n}$ at $\mathrm{Od}=0$. 
In Figure $7 \mathrm{a}$, the viscous drag coefficient increases with the angle from a minimum at $0^{\circ}$ to a maximum at $90^{\circ}$.
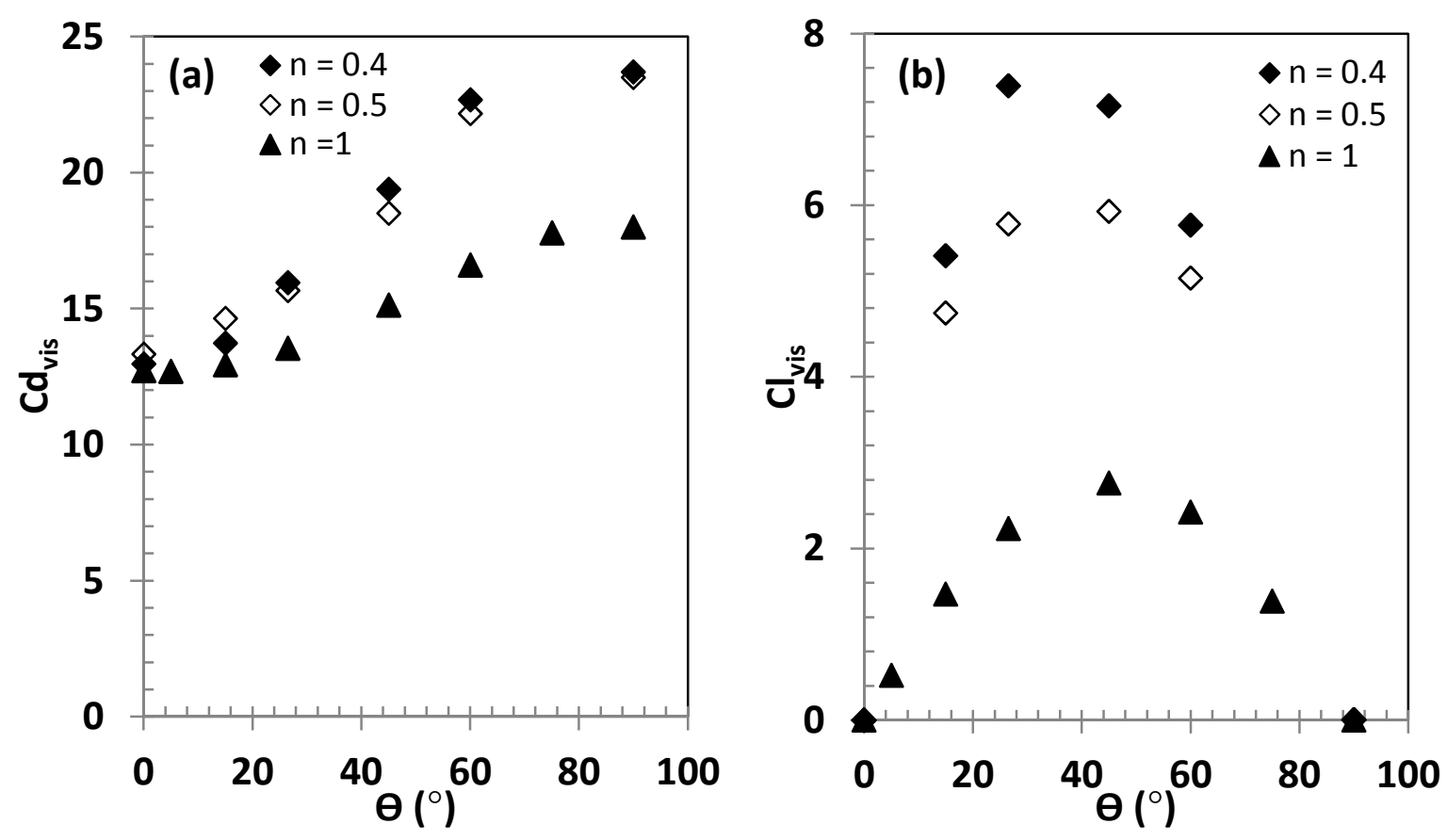

Figure 7: Variation in viscous drag (a) and lift (b) as a function of the angle for a Reynolds number $\mathrm{Re}=0.001$, a gap $\mathrm{G}=3$ and for various values of $n$.

As shear thinning increases i.e. when $\mathrm{n}$ decreases, the intensity of the viscous drag coefficient increases. The Newtonian values are always the smallest. The effect of shear thinning is weak at small angles. It is maximum at the $90^{\circ}$ angle.

Figure $7 \mathrm{~b}$ shows that, as $\mathrm{n}$ decreases, viscous lift $\mathrm{Cl}_{\text {vis }}$ increases. It is also found that maximum lift measured at the $45^{\circ}$ angle for a Newtonian fluid $(\mathrm{n}=1$, Od $=0$ ) occurs at smaller angles when $\mathrm{n}$ decreases. From $\mathrm{n} \leq 0.4$, the maximum lift is found at $26.5^{\circ}$.

\subsubsection{Yield stress fluid}

Figure 8 shows the influence of $\mathrm{n}$ on the plastic coefficients $\mathrm{Cd}^{*}$ and $\mathrm{Cl}^{*}$ as a function of the inclination angle for a gap $\mathrm{G}=3$ with the Oldroyd number Od $=5.5$ and 179 . 
Regardless of the value of $\mathrm{Od}$, when the inclination angle $\theta$ increases, the plastic drag coefficient $\mathrm{Cd}^{*}$ increases as well. The increase in this drag coefficient depends on the intensity of Od. At the same angle, the drag coefficient values are greater when Od decreases, as shown in Figs 8a and 8b. In fact, viscous stresses increase and add to the yield stresses, thus increasing the shear forces acting on the plate.

Concerning the influence of the shear-thinning index $n$, the increase in $n$ leads to an increase in the drag coefficient as shown in Figure 8a. The effect of the shear-thinning index on the drag, as shown in Figure 8b, is thus reduced when Od increases.

The asymptotic values of theoretical plastic drag at the $0^{\circ}$ and $90^{\circ}$ angles given respectively by Piau and Debiane ${ }^{8}$ and by Merifield et al. ${ }^{46}$ are shown in Figure 8 . For an infinitely thin plate in an infinite domain, and parallel to the flow, Piau and Debiane ${ }^{8}$ proposed the following expression for plastic drag:

$\mathrm{Cd}^{*}=4\left(1+\left(\left[\frac{2}{\pi} \frac{1+\mathrm{n}}{\mathrm{n}}\right]^{\mathrm{n} /(1+\mathrm{n})} \frac{1}{\mathrm{Od}}\right)^{1 /(1+\mathrm{n})}\right)$ 
Figure 8 shows that the difference with our results is substantial (46\%) especially for small Od values. This is due to the fact that Piau and Debiane's theory is based on an infinitely thin plate, which is not the case in this study $(\mathrm{e} / \mathrm{L}=0.1)$. With a much smaller thickness (e/L=0.003), a difference of less than $7 \%$ was obtained. If the results of Patel and Chhabra ${ }^{16}$ for an elliptical cylinder of the greatest slenderness used $(E=10)$, and for a Bingham fluid, are applied, a value of $\mathrm{Cd}^{*}=6+\frac{4.83}{\mathrm{Od}}+\frac{1.82}{\mathrm{Od}^{0.532}}$ is found. Formula (20) and table 11 of the Patel and Chhabra publication ${ }^{16}$ were used with our length scale. The flow for a high aspect ratio $\mathrm{E}$ tends towards a configuration corresponding to a plate placed parallel to the flow. Note that the slenderness E used by Patel and Chhabra is the inverse of the ratio e/L used in our study so for $\mathrm{E}=10$ then $\mathrm{e} / \mathrm{L}=0.1$. For $\mathrm{Od}=5.5$ a value of $\mathrm{Cd}^{*}=7.6$ is obtained and for $\mathrm{Od}=179, \mathrm{Cd}^{*}=6.11$. These predictions are relatively close to the numerical solution and better than those of Piau and Debiane ${ }^{8}$.

For a high Od value $(\mathrm{Od}=179)$, Patel and Chhabra's solution is satisfactory, among other things because the aspect ratio is close to that of the numerical simulation. 
Looking at Patel and Chhabra's predictions, ${ }^{16}$ a value of $\mathrm{Cd}^{*}=20.87+\frac{28}{\mathrm{Od}^{0.582}}$ is obtained for $\mathrm{E}=0.1$, with their formula (20) and their table 11 . For $\mathrm{Od}=5.5$ and $\mathrm{Od}=179$ values of $\mathrm{Cd}^{*}=$ 31.25 and $\mathrm{Cd}^{*}=22.23$ are obtained respectively. If these values are placed on Figure 8a, very good agreement can be seen with the computed numerical solutions of our study.
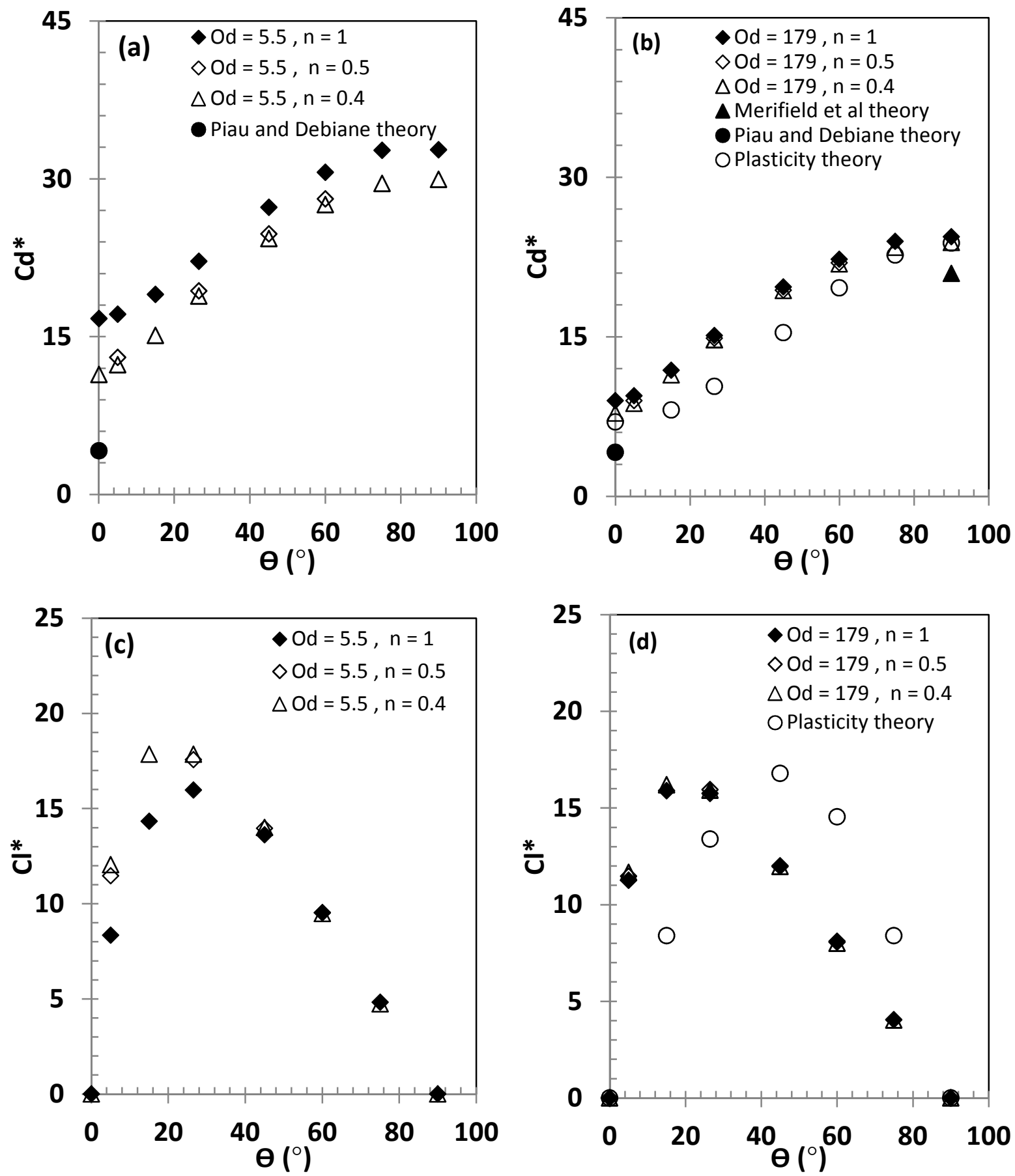

Figure 8: Variation in plastic drag (a) and (b), lift coefficient (c), (d) as a function of the inclination angle for two Oldroyd numbers values $\mathrm{Od}=5.5$ and $\mathrm{Od}=179$ with $\mathrm{n}=1$ and $\mathrm{n}=0.4$ at gap $\mathrm{G}=3$. 
The plasticity theory that led to eqs. (12) - (15) can also be applied. As can be seen in Figure $8 \mathrm{~b}$, a satisfactory approximation was obtained with these results.

Figures $8 \mathrm{c}$ and $8 \mathrm{~d}$ show the variation in plastic lift coefficient as a function of the inclination angle for $\mathrm{Od}=5.5$ and 179 . The lift is zero for $0^{\circ}$ and $90^{\circ}$ angles and is maximum at a particular angle. In Figure 8c, the maximum lift is obtained at $\theta=26.5^{\circ}$ for $n=1$. This maximum is subsequently located between $\theta=15^{\circ}$ and $\theta=26.5^{\circ}$ for $n=0.5$ and $n=0.4$ when the Oldroyd number Od is 5.5. Thus, when the Oldroyd number Od increase to a high value $(\mathrm{Od}=179)$ in Figure $8 \mathrm{~d}$, the maximum lift is obtained between $\theta=15^{\circ}$ and $\theta=26.5$ regardless of $\mathrm{n}$. This inclination angle corresponding to the maximum lift is different to that obtained by the plasticity theory of eq. (13) $\left(\theta=45^{\circ}\right)$.

Our numerical results approximate the results of Patel and Chhabra ${ }^{17}$ relating to the flow of a Bingham fluid around a cylinder inclined at an angle to the direction of flow. They find that the amplitude of the lift coefficient is maximun for an angle of about $30^{\circ}$ (26.5 ${ }^{\circ}$ in our study). There is a degree of imprecision because their results are calculated every $15^{\circ}$. As in our study, this maximum does not seem to be significantly influenced by the value of Od. The orders of magnitude of $\mathrm{Cd} *$ and $\mathrm{Cl} *$ cannot be compared because their aspect ratio is very different from that of our study.

When the yield stress is high $(\mathrm{Od}=179)$, the curves no longer depend on $\mathrm{n}$ because the viscous effects are negligible. This variation as a function of $n$ is different in the case of a shear-thinning fluid (Fig. 7b) and when the yield stress $(\mathrm{Od}=5.5)$ is low (Fig. 8c). 
Additional calculations were made to determine the variation in plastic drag and plastic lift for a value of $\mathrm{Od}=1000$ and for a given angle $\left(\theta=26.5^{\circ}\right) . \mathrm{Cd}^{*}$ values of 14.73 and 14.6 were obtained for $\mathrm{Od}=179$ and $\mathrm{Od}=1000$ respectively. The difference between these two values is $0.88 \%$. The values of lift $\mathrm{Cl}^{*}$ are of the order of 15.92 and 15.763 respectively for $\mathrm{Od}=179$ and $\mathrm{Od}=1000$. The difference between these two values is $0.9 \%$. In short, the increase in yield stress effects for $\mathrm{Od}=179$ or more becomes negligible on the plastic drag and lift values since the values of $\mathrm{Cd}^{*}$ and $\mathrm{Cl}^{*}$ for $\mathrm{Od}=179$ and $\mathrm{Od}=1000$ are very close. This trend obtained on the values of $\mathrm{Cd}^{*}$ and $\mathrm{Cl}^{*}$ for these two Od values remains valid, regardless of the angle. The values of $\mathrm{Cd}^{*}$ and $\mathrm{Cl}^{*}$ for very high $\mathrm{Od}$ values correspond to the situation where the plate is motionless in the fluid. These constant values can be used to calculate the minimum yield stress to keep the plate static in the fluid. This aspect will be discussed later in this article.

The influence of domain size was also studied up to $G=100$ in order to check whether the wall effects are negligible with respect to the values of drag and lift at high Od values $(\mathrm{Od}=$ $179)$ and at lower Od values $(\mathrm{Od}=5.5)$ for $\mathrm{n}=1$ and $\mathrm{n}=0.4$ and for $\theta=26.5^{\circ}$.

The maximum difference obtained on the values of $\mathrm{Cd}^{*}$ and $\mathrm{Cl}^{*}$ between the gap $\mathrm{G}=1$ and $\mathrm{G}$ $=100$ was $3.5 \%$. From $\mathrm{G}=1$ and $\mathrm{Od}=5.5$, the domain can be considered as infinite, which means without any interaction effect with the walls. Other complementary studies of the influence of the gap $\mathrm{G}$ at $90^{\circ}$ have also confirmed that wall effects on the plastic drag are negligible from a gap $\mathrm{G}=1$. For example, a difference of $1 \%$ was found between plastic drag values for a gap $\mathrm{G}=1$ and a gap $\mathrm{G}=3$ for $\mathrm{Od}=5.5$. 
In addition, as previously shown, the values of $\mathrm{Cd}^{*}{ }_{\infty}$ and $\mathrm{Cl}_{\infty}^{*}$ can be used to estimate the minimum value of the yield stress needed to keep the plate static in the fluid. In this case, the force created by the weight of the plate (mass $(\mathrm{m}) \times$ constant gravity $(\mathrm{g})$ ) is balanced by the force exerted by the yield stress flow. The yield stress needed to maintain the motionless

plate can be defined by: $\tau_{0} \geq \frac{\mathrm{m} . \mathrm{g}}{\mathrm{Cd}^{*} \text {.L.l }}$. Thus, the yield stress required is inversely proportional to $\mathrm{Cd}^{*}{ }_{\infty}$. The results from Figure 8 therefore show that when the inclination angle increases, a smaller yield stress is required to stabilize it in suspension.

\section{Numerical and experimental comparison}

\subsection{Newtonian fluid}

As previously seen, $\mathrm{Wu}$ and Thompson ${ }^{6}$ proposed laws (eqs. (8) and (9)) which can be used to calculate the force coefficients for a creeping flow. The drag coefficient values of the plate at $0^{\circ}$ and $90^{\circ}$ angles introduced in eqs. (8) and (9) to calculate Newtonian theory are derived from the numerical values for a gap $G=3$.

Figure 9 shows a comparison of the numerical and experimental results as well as the Newtonian theory of viscous drag and lift coefficient as a function of angle for a Newtonian fluid. Figure 9a shows the increase in viscous drag when the angle increases. The maximum difference between the numerical and experimental results was $6 \%$. By taking into account the maximum experimental uncertainties, estimated at $15 \%$, the values are similar. Good agreement was found between Newtonian theory (eq. (8)) and the experimental results. 
Figure $9 \mathrm{~b}$ shows a similar change in lift between the experimental measurements, the numerical prediction and Newtonian theory of eq. (9). In particular, the maximum lift is obtained for $\theta=45^{\circ}$. The maximum differences obtained are of the order of $17 \%$. When the experimental uncertainties are taken into account, a good correlation is observed between experimental measurements, numerical predictions and Newtonian theory.
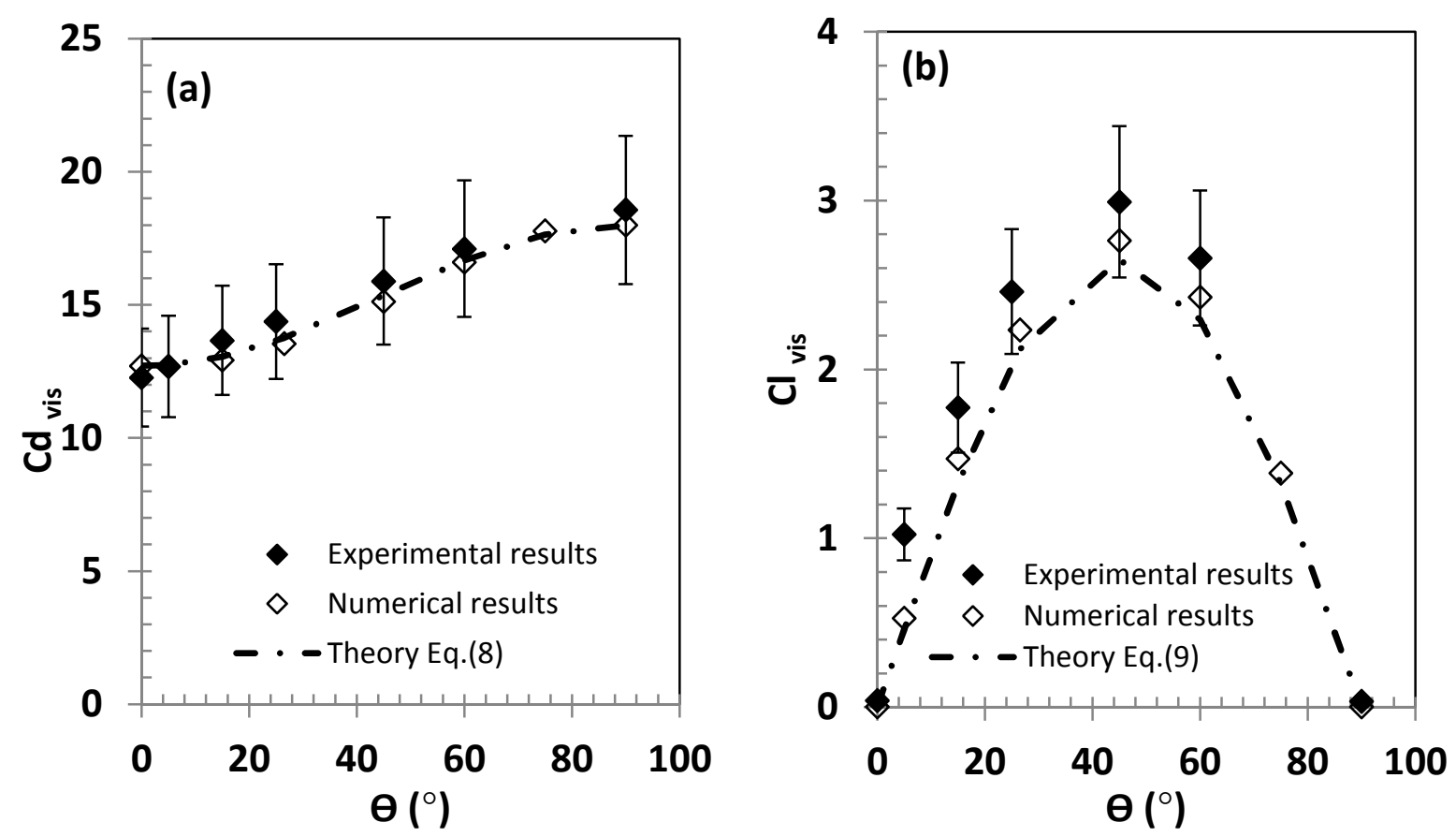

Figure 9: Variation in viscous drag (a) and lift (b) coefficients as a function of the inclination angle for a Newtonian fluid with negligible inertia and a gap $G=3$.

\subsection{Yield stress fluid}

The experimental drag and lift measurements will now be compared with the numerical simulation results using the Herschel-Bulkley model (Figure 10) for Oldroyd number values of $\mathrm{Od}=5.5$ and 179 . 

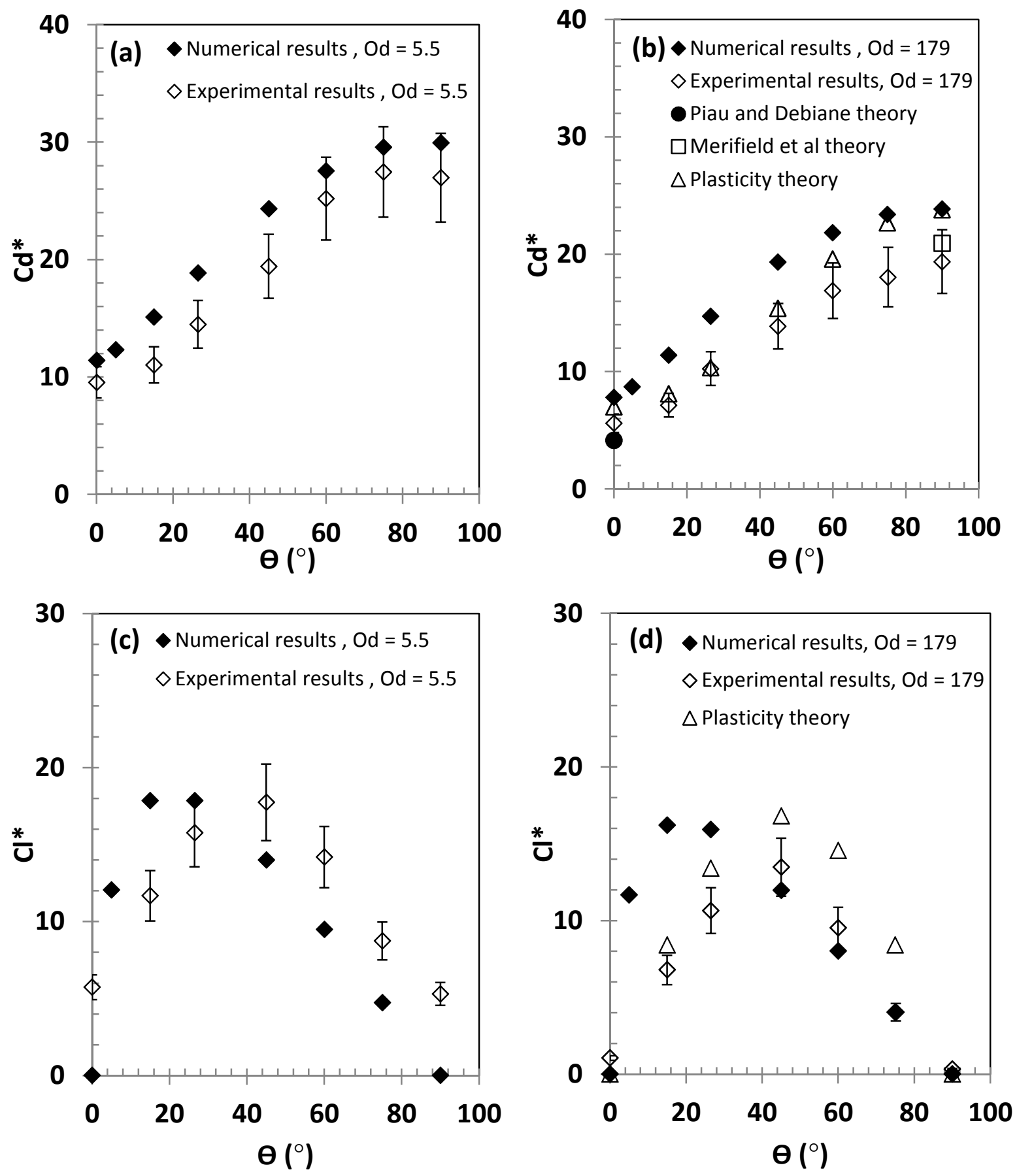

Figure 10: Variation in plastic drag (a) and (b), and plastic lift (c) and (d) coefficients as a function of angle for Oldroyd numbers $\mathrm{Od}=5.5$ and 179 , with a gap $\mathrm{G}=3$.

The numerical values of the drag coefficients are generally slightly higher than experimental results, but follow the same variations. For the plastic lift (Figures 10c and 10d), the numerical results are greater than the experimental results over the range $5^{\circ}$ to $26.5^{\circ}$. However, from the $45^{\circ}$ angle, the experimental results are greater than the numerical results. 
This angle of $45^{\circ}$ corresponds to the maximum of the measured lift. Numerically it is smaller and depends on the yield stress value Od and the shear-thinning index n. As shown previously in the numerical results study, the effect of mesh type and plate shape on the drag and lift values is negligible. Any possible numerical singularity of the corners is absent.

We propose to discuss the differences between experimental results and anelastic viscoplastic modeling by referring to the recent publication of Fraggedakis et al. $^{37}$. These authors numerically modeled flows during sedimentation of a sphere in a yield stress fluid and compared their numerical predictions with experiments in Carbopol gels. The constitutive law describes the elastic effects and the hardening effects observed on Carbopol gels by Dimitriou et $\mathrm{al}^{23}$. They found that the plastic drag $\mathrm{Cd}^{*}$ values decreased when the elasticity of the material increased. This could explain the differences between our numerical and experimental results. However, in our study, the dimensionless Deborah number used to characterize the elasticity of a material is very small. This number can be defined by: De = $\frac{\mathrm{U}}{\mathrm{L}} \cdot \mathrm{K}^{1 / \mathrm{n}}$. In this study, it varies between $1.274 \times 10^{-4}$ and $1.33 \times 10^{-4}$. The elastic effects therefore seem negligible. On the other hand, this study focuses on high plastic effects with Oldroyd numbers ranging from 5.5 to 179 . The phenomena of this study seem more accentuated by plasticity rather than by elasticity. This would be consistent with the analysis proposed by Fraggedakis et al. ${ }^{37}$. In their study, they introduced the concept of isotropic kinematic hardening (IKH) proposed by Dimitriou et al. ${ }^{23}$. The idea of this model is that the yield stress surface of the material changes dynamically with the flow field. Fraggedakis et al. ${ }^{37}$ showed that this concept introduced in numerical modeling can be used to represent the experimental results obtained with Carbopol gels. To better understand the shift in the maximum lift, it would certainly be interesting to integrate in the numerical study models describing precisely the solid-liquid transition like the IKH model. Moreover, the influence of 
initial residual stresses in the gel cannot be excluded. Mougin et al. ${ }^{47}$ have shown the influence of these stresses in a study where they analyzed the rise of a bubble in Carbopol gels under quasi-static flow. In addition, Ahonguio et al. ${ }^{29}$ showed the variation in normal forces for these types of fluid as a function of the shear rate. The presence of these normal forces could in part explain the differences obtained.

\section{Conclusion}

The influence of plate inclination angle in the non-inertia flow of a yield fluid and a Newtonian fluid was studied experimentally and numerically. For yield stress fluids, the study focused on predominant plasticity effects.

From a numerical point of view, the variation in drag and lift coefficients as a function of the inclination angle, the shear-thinning index and the stress yield was established.

A good correlation for Newtonian fluids was found for the drag and lift coefficient as a function of angle between the experimental and numerical results as well as a good approximation with the prediction of the $\mathrm{Wu}$ and Thompson ${ }^{6}$ model.

For yield stress fluids, the comparison between numerical prediction and experimental measurements shows that the variation in lift and drag as a function of angle is similar. However, the maximum lift is predicted by the plasticity theory and experimental measurements at the $45^{\circ}$ angle, whereas numerical predictions based on the Herschel-Bulkley model give a lower angle.

Analytical solutions of plastic drag and lift coefficients from the plasticity theory or boundary layer on a flat plate predict the experimental measurements relatively well. The numerical solutions give results that are relatively close to the experimental measurements except for the maximum lift angle. 
The differences between experimental and numerical results do not seem to derive from elastic effects, but rather from phenomena related to plasticity. The change in yield stress or the isotropic kinematic hardening proposed by Dimitriou et al. ${ }^{2}$ would certainly appear to play a role in these differences. In the future, modeling should take into account this more realistic solid-liquid transition for Carbopol gels.

\section{Acknowledgments}

The Laboratoire Rhéologie et Procédés is part of the LabEx Tec 21 (Investissements d'Avenir - grant agreement $\mathrm{n}^{\circ}$ ANR-11-LABX-0030) and of the PolyNat Carnot Institute (Investissements d'Avenir - grant agreement nºANR-11-CARN-030-01).

The PhD Research project of Z. Ouattara has been made possible thanks to an Ivory Coast Scholarship.

\section{REFERENCES}

1. In KM, Choi DH, Kim M-U. Two-dimensional viscous flow past a flat plate. Fluid Dynamics Research. 1995;15(1):13-24.

2. Tamada K, Miura H, Miyagi T. Low-Reynolds-number flow past a cylindrical body. Journal of Fluid Mechanics. 1983;132(1):445.

3. Janssen E. Flow past a flat plate at low Reynolds numbers. Journal of Fluid Mechanics. 1958;3(04):329.

4. Tomotika S, Aoi T. The steady flow of a viscous fluid past an elliptic cylinder and a flat plate at small Reynolds numbers. The Quarterly Journal of Mechanics and Applied Mathematics. 1953;6(3):290-312. 
5. Hudson JD, Dennis SCR. The flow of a viscous incompressible fluid past a normal flat plate at low and intermediate Reynolds numbers: the wake. Journal of Fluid Mechanics. 1985;160(1):369.

6. Wu J, Thompson MC. Non-Newtonian shear-thinning flows past a flat plate. Journal of non-newtonian fluid mechanics. 1996;66(2-3):127-144.

7. Piau J-M. Viscoplastic boundary layer. Journal of non-newtonian fluid mechanics. 2002;102(2):193-218.

8. Piau J-M, Debiane K. The adhesive or slippery flat plate viscoplastic boundary layer for a shear-thinning power-law viscosity. Journal of Non-Newtonian Fluid Mechanics. 2004;117(2-3):97-107.

9. Boujlel J, Maillard M, Lindner A, Ovarlez G, Chateau X, Coussot P. Boundary layer in pastes-Displacement of a long object through a yield stress fluid. Journal of Rheology. 2012;56(5):1083-1108.

10. N. J. Balmforth, R. V. Craster, D. R. Hewitt, S. Hormozi, and A. Maleki. Viscoplastic boundary layers. J. Fluid Mech. (2017), vol. 813, pp. 929-954.

11. Ahonguio F, Jossic L, Magnin A, Dufour F. Flow of an elasto-viscoplastic fluid around a flat plate: Experimental and numerical data. Journal of Non-Newtonian Fluid Mechanics. 2016;238:131-139.

12. Ferreira MR, Furtado GM, Hermany L, Frey S, Naccache MF, de Souza Mendes PR. External flows of elasto-viscoplastic materials over a blade. Proceedings of the ENCIT. 2014;15:10-13.

13. Brookes, G. F., and R. L. Whitmore. Drag forces in Bingham plastics. Rheol. Acta, 8, 472. 1969.

14. Brookes, G. F, and R. L. Whitmore. The Static Drag on Bodies in Bingham Plastics. Rheol. Acta, 7, 189. 1968.

15. Savreux F, Jay P, Magnin A. Flow normal to a flat plate of a viscoplastic fluid with inertia effects. AIChE Journal. 2005;51(3):750-758.

16. Patel SA, Chhabra RP. Steady flow of Bingham plastic fluids past an elliptical cylinder Journal of Non-Newtonian Fluid Mechanics, 2013; 202 :32-53

17. Patel SA, Chhabra RP. Effect of the angle of incidence on laminar forced convection from an elliptical cylinder in Bingham plastic fluids. Numerical Heat Transfer, Part A: Applications, 2016; 70(8):917-937 
18. R. S. Merifield, S. W. Sloan, and H. S. Yu. Stability of plate anchors in undrained clay. (2001). Géotechnique 51, No. 2, 141 153 .

19. Bemben SM, Kalajian EH, Kupferman MM, others. The vertical holding capacity of marine anchors in sand and clay subjected to static and cyclic loading. In: Offshore Technology Conference. Offshore Technology Conference; 1973. https://www.onepetro.org/conference-paper/OTC-1912-MS. Accessed October 13, 2017.

20. Ovarlez G, Barral Q, Coussot P. Three-dimensional jamming and flows of soft glassy materials. Nature Materials. 2010;9:115-119.

21. Martinie L., Buggisch H., Willenbacher N. Extending yield-stress fluid paradigms. $J$. Rheol. ,2013,57, 627-646.

22. Piau JM. Carbopol gels: Elastoviscoplastic and slippery glasses made of individual swollen sponges. Journal of Non-Newtonian Fluid Mechanics. 2007;144(1):1-29.

23. Dimitriou CJ, Ewoldt RH, McKinley GH. Describing and prescribing the constitutive response of yield stress fluids using large amplitude oscillatory shear stress (LAOStress). Journal of Rheology. 2013;57(1):27-70.

24. Goodrich B. Product Literature. Cleveland; 1997.

25. Magnin A, Piau JM. Cone-and-plate rheometry of yield stress fluids. Study of an aqueous gel. Journal of Non-Newtonian Fluid Mechanics. 1990;36:85-108.

26. Magnin A, Piau JM. Shear rheometry of fluids with a yield stress. Journal of NonNewtonian Fluid Mechanics. 1987;23:91-106.

27. Meeker SP, Bonnecaze RT, Cloitre M. Slip and Flow in Soft Particle Pastes. Physical Review Letters. 2004;92(19).

28. P. Coussot, H. Tabuteau, X. Chateau, L. Tocquer, and G. Ovarlez. Aging and solid or liquid behavior in pastes. Journal of Rheology 50, 975 (2006).

29. Ahonguio F, Jossic L, Magnin A. Influence of surface properties on the flow of a yield stress fluid around spheres. Journal of Non-Newtonian Fluid Mechanics. 2014;206:5770.

30. Yarin AL, Zussman E, Theron A, Rahimi S, Sobe Z, Hasan D. Elongational behavior of gelled propellant simulants. Journal of Rheology. 2004;48(1):101-116.

31. Balmforth NJ, Dubash N, Slim AC. Extensional dynamics of viscoplastic filaments: I. Long-wave approximation and the Rayleigh instability. Journal of Non-Newtonian Fluid Mechanics. 2010;165(19-20):1139-1146. 
32. Balmforth NJ, Dubash N, Slim AC. Extensional dynamics of viscoplastic filaments: II. Drips and bridges. Journal of Non-Newtonian Fluid Mechanics. 2010;165(19-20):11471160.

33. Lidon P, Villa L, Manneville S. Power-law creep and residual stresses in a carbopol gel. Rheologica Acta. 2017;56(3):307-323.

34. F. Caton and C. Baravian. Plastic behavior of some yield stress fluids:From creep to long-time yield. Rheol. Acta 47, 601-607 (2008).

35. Divoux T, Tamarii D, Barentin C, Manneville S. Transient Shear Banding in a Simple Yield Stress Fluid. Physical Review Letters. 2010;104(20).

36. T.Divoux,C.Barentin, and S.Manneville. From stress-induced fluidization processes to Herschel-Bulkley behaviour in simple yield stress fluids. Soft Matter 7, 8409-8418 (2011).

37. Fraggedakis D, Dimakopoulos Y, Tsamopoulos J. Yielding the yield-stress analysis: a study focused on the effects of elasticity on the settling of a single spherical particle in simple yield-stress fluids. Soft Matter. 2016;12(24):5378-5401.

38. Merkak O, Jossic L, Magnin A. Spheres and interactions between spheres moving at very low velocities in a yield stress fluid. Journal of Non-Newtonian Fluid Mechanics. 2006;133(2-3):99-108.

39. Tokpavi DL, Jay P, Magnin A, Jossic L. Experimental study of the very slow flow of a yield stress fluid around a circular cylinder. Journal of Non-Newtonian Fluid Mechanics. 2009;164(1-3):35-44.

40. Raghavendra, Dhanvi Vengre. Performance of Plate Anchors. Master's thesis, Texas A \& M University. (2016)

41. Aubeny C.P., Chi C. Mechanics of drag embedment anchors in a soft seabed. Journal of Geotechnical and geoenvironmental engineering, 2010, 136,57-68.

42. Anderson, K. H., Murff, J. D., and Randolph, M. R. Deepwater Anchor Design PracticeVertically Loaded Drag Anchors (2003). Phase II Report to API/Deepstar, Norwegian Geotechnical Institute, Norway, Offshore Technology Research Center, USA and Centre for Offshore Foundation Systems, Australia.

43. Papanastasiou TC. Flows of Materials with Yield. Journal of Rheology. 1987;31(5):385404.

44. Tokpavi DL, Magnin A, Jay P. Very slow flow of Bingham viscoplastic fluid around a circular cylinder. Journal of Non-Newtonian Fluid Mechanics. 2008;154(1):65-76. 
45. Mitsoulis E. On creeping drag flow of a viscoplastic fluid past a circular cylinder: wall effects. Chemical Engineering Science. 2004;59(4):789-800.

46. Merifield RS, Lyamin AV, Sloan SW, Yu HS. Three-dimensional lower bound solutions for stability of plate anchors in clay. Journal of Geotechnical and Geoenvironmental Engineering. 2003;129(3):243-253.

47. Mougin N, Magnin A, Piau JM. The significant influence of internal stresses on the dynamics of bubbles in a yield stress fluid. Journal of Non-Newtonian Fluid Mechanics. 2012; 171-172: 42-55.

48. Atapattu DD, Chhabra RP, Uhlherr PHT. Wall effect for spheres falling at small Reynolds number in a viscoplastic medium. Journal of Non-Newtonian Fluid Mechanics, 1990; 38(1):31-42

49. Hariharaputhiran M, Subramanian RS, Campbell GA, Chhabra RP. The settling of spheres in a viscoplastic fluid. Journal of Non-Newtonian Fluid Mechanics, 1998; 79(1):87-97

50. Burgos GR, Alexandrou AN, Entov V. On the determination of yield surfaces in Herschel-Bulkley fluids. Journal of Rheology. 1999; 43(3):463. 